\title{
Aumentar receitas ou cortar gastos? Discutindo o nexo entre receitas e despesas do governo central brasileiro
}

\author{
Jevuks Matheus Araujo \\ Professor - Universidade Federal da Paraíba (UFPB) \\ Endereço: Cidade Universitária - João Pessoa/PB \\ CEP: 58051-900 - E-mail: jevuks@gmail.com
}

\section{Rozane Bezerra Siqueira}

Professor - Universidade Federal de Pernambuco (UFPE)

Endereço: Av. Moraes Rego, 1235 - Cidade Universitária

CEP: 50670-901 - E-mail: rozane_siqueira@yahoo.com.br

\section{Cassio Nobrega Besarria}

Professor - Universidade Federal da Paraíba (UFPB)

Endereço: Cidade Universitária - João Pessoa/PB

CEP: 58051-900 - E-mail: cassiodanobrega@yahoo.com.br

Recebido: 01/09/2016. Aceite: 26/06/2017

\section{Resumo}

O objetivo deste estudo é analisar a relação intertemporal de curto e longo prazo entre as variáveis gasto e receita do governo federal brasileiro. Usando dados mensais de 1997 a 2015, o estudo aplica técnicas de cointegração e estima modelos de correção de erro assimétrico (MCE-TAR e MCE-MTAR). Como análise de robustez foi realizada uma simulação de choques nas variáveis receitas e despesas do governo central. Os resultados mostram que o governo respeita sua restrição orçamentária apenas para o período de 1997 a 2013. Nesse período há evidências, no curto prazo, de uma relação causal unidirecional do tipo tax-spend, na qual uma redução de imposto gera aumento no gasto e no longo prazo a relação causal é bidirecional. Não foi possível, ao contrário de estudos anteriores, identificar evidências de uma relação causal do tipo spend-tax. Os resultados também refutaram a interpretação tradicional de que o caminho para o controle do gasto é reduzir a arrecadação do governo. De forma geral, os resultados mostram que ao incorporar na análise os anos de 2014 e 2015, o governo central deixa de respeitar a sua restrição orçamentária, e não é possível estabelecer uma relação causal entre receita e gasto governamental.

\section{Palavras-Chave}

Receita. Despesa. Ilusão fiscal. Modelos de Correção de Erro.

\footnotetext{
- Agradecemos aos pertinentes comentários e sugestões dos pareceristas anônimos. Além desses, somos gratos aos debatedores Alexandre Manoel Ângelo da Silva, José Ricardo Bezerra Nogueira, Jocildo Bezerra Fernandes e Paulo Amilton Maia Leite Filho. Erros ainda existentes são de responsabilidade dos autores.
} 


\begin{abstract}
The aim of this study is to analyze the short and long term intertemporal relationship between spending and revenue variables of the Brazilian federal government. Using monthly data 1997 to 2015 the study applied cointegration techniques and estimates asymmetric error correction models (ECM-TAR and ECM-MTAR). As a robustness analysis a simulation of shock was performed on variable revenue and expenditure of the central government. The results show that the government respects its budget constraint only for the period from 1997 to 2013. During this period there is evidence, in the short term, of a one-way causal relationship type tax-spend, where a tax reduction generates an increase in spending and the long-term causal relationship is bidirectional. Could not unlike previous studies, identify evidence of a causal relationship of spend-tax type. The results also disproved the traditional interpretation that the way to control spending is to reduce government revenues. Overall, the results show that by incorporating the analysis of the years 2014 and 2015 the central government fails to respect their budget constraint and cannot establish a causal relationship between income and government spending.
\end{abstract}

\title{
Keywords
}

Revenue. Expenditure. Fiscal illusion. Error Correction Models.

\section{JEL Classification}

H2O. H50. H6O.

\section{Introdução}

O debate sobre a relação intertemporal entre receita e gasto do governo teve início nos anos 30 do século passado e se intensificou em meados dos anos 80 , motivado pelo crescimento do déficit orçamentário norte-americano. A importância da análise da interação entre receitas e gastos do Governo Federal está fortemente ligada a adoção de políticas de correção do déficit orçamentário e sustentabilidade da dívida (Payne 2003).

Niskanen (2006) observa que o principal argumento da época era o defendido pelos republicanos. Segundo o autor, para esses a política para controle dos gastos governamentais deveria ser baseada no corte de impostos (starve the beast). Nesse sentido, o discurso republicano afirmava que os impostos (receita) geram gastos (tax-spend) com uma relação positiva. Essa discussão se fortaleceu quando Friedman (1978) passou a defender as leis que limitam os gastos governamentais, argumentando que é preciso cortar os impostos para conter a expansão dos gastos. Para Friedman (1978), a redução da receita deve conter os gastos, uma vez que os legisladores com 
limites mais rígidos de orçamento devem privilegiar as demandas gerais da população em detrimento das demandas de pequenos grupos especiais.

Um corte permanente de impostos é a medida mais eficaz para conter os gastos governamentais. A redução dos impostos reduz o gasto governamental e ainda pode aumentar a riqueza e a renda das pessoas. O único caminho para controlar a expansão do gasto é cortar imposto, em outras palavras, deve-se punir o filho esbanjador cortando a sua mesada (Friedman 2003). No entanto, a hipótese de relação causal do tipo receita gera gasto (tax-spend) não é explicada apenas pelo argumento de corte de impostos para redução dos gastos (starve the beast). Outra explicação, factível e contrária ao argumento de Friedman (1978 e 2003), é que se existe uma relação causal do tipo receita gera gasto, ela é negativa (inversa), podendo ser representada pelo fato de um corte/aumento nos impostos aumentar/ reduzir os gastos.

O argumento da relação negativa (inversa) está associado à existência de ilusão fiscal. Buchanan e Wagner (1977) argumentam que o governo usa diferentes formas de financiamento do gasto público e que um corte em impostos não reduz os gastos. O nível do gasto é mantido por outros mecanismos fiscais, tais como: imposto inflacionário e expansão da dívida. O corte de impostos gera uma mudança na percepção dos preços dos bens públicos, ou seja, os contribuintes passam a acreditar que houve uma redução no preço dos bens e serviços públicos ofertados pelo governo.

Partindo do pressuposto de que os impostos são compreendidos como o preço dos bens públicos, Niskanen (2006) ressalta que o argumento de Friedman (1978 e 2003) é teoricamente implausível, pois a redução de impostos tem o mesmo efeito de um controle de preços, ou seja, deve aumentar a quantidade demandada pelos bens e serviços ofertados pelo governo. Desta forma, a interpretação é de uma simples curva de demanda em que os gastos possuem uma relação inversa com os impostos.

Trazendo novos elementos à discussão, Barro (1974, 1979) desenvolveu um modelo de determinação da dívida pública, também conhecido na literatura econômica como teorema da equivalência ricardiana. Esse mostrou que o financiamento do gasto público por emissão de dívida tem os mesmos efeitos (sobre a demanda e a taxa de juros) de um financiamento por impostos. Na visão do autor, os consumidores responderão ao aumento futuro dos impostos com elevação da poupança presente. Desta 
forma, o financiamento do gasto público por emissão dívida é equivalente ao financiamento por impostos. O modelo desenvolvido por Barro (1974, 1979) induz a uma terceira hipótese de relação causal entre impostos e gastos, em que o aumento nos gastos gera um aumento dos impostos (spendtax). Neste contexto, Payne (2003) observa que o endividamento presente do governo resultará em passivos futuros; assim, o aumento de impostos é uma resposta à expansão no gasto público.

A teoria de ilusão fiscal sugere uma relação positiva entre gastos e dívida. Dessa forma, a validação da teoria de ilusão fiscal exclui a possibilidade de verificação do teorema da equivalência proposto por Barro (1974 e 1979). Segundo a teoria de ilusão fiscal, a diluição dos custos dos bens e serviços ofertados por longos períodos distorce a percepção do contribuinte com relação ao preço pago e a quantidade de bens e serviços consumidos. $\mathrm{O}$ financiamento por dívida faz com que os contribuintes subestimem as obrigações fiscais e acreditem estarem mais ricos do que de fato estão. Desta forma, os contribuintes são menos resistentes à expansão do gasto público. Segundo Ricardo (1821), o sistema de financiamento dos gastos governamentais por emissão de dívida cria sobre os contribuintes a ilusão de serem mais ricos do que verdadeiramente são. Ricardo (1821, cap. 17, 179) afirma que o financiamento por dívida é "um sistema que tende a nos tornar menos poupadores e a nos cegar sobre a nossa real situação".

Ademais, ressalta-se a hipótese da relação entre receitas e gastos também conhecida como sincronização, em que essa mostra das decisões do governo sobre o montante de receita e gasto são realizadas de forma simultânea. Assim, a relação causal entre as variáveis é bidirecional. Essa hipótese foi tratada por Meltzer e Richard (1981), e esses mostraram que o tamanho do governo é determinado pela escolha de maximização do bem-estar do indivíduo decisivo, representado, em uma democracia, pelo eleitor mediano. Para esses, os eleitores não sofrem de ilusão fiscal e, antes de escolherem o nível ótimo de despesa e receita, observam os benefícios e custos marginais dos programas de governo. Assim, a possibilidade de equilíbrio entre gastos e receitas governamentais não pode ser descartada. Por fim, há a hipótese de independência institucional, a qual sugere a receita e a despesa do governo são independentes uma da outra. Dessa forma, não existe relação causal entre as variáveis. Segundo Baghestani e Mcnown (1994), a independência institucional resulta da falta concordância entre os setores governamentais responsáveis pelas escolhas de arrecadação e execução das despesas. 
Nos últimos 25 anos, a economia brasileira passou por importantes transformações. As mudanças ocorridas na política fiscal são facilmente percebidas pela evolução do resultado primário do governo federal (Gráfico 1). Observa-se que no início dos anos noventa o resultado primário apresentava fortes oscilações, resultante da instabilidade do nível de preços da economia que exercia forte influência sobre o fluxo de caixa do governo federal.

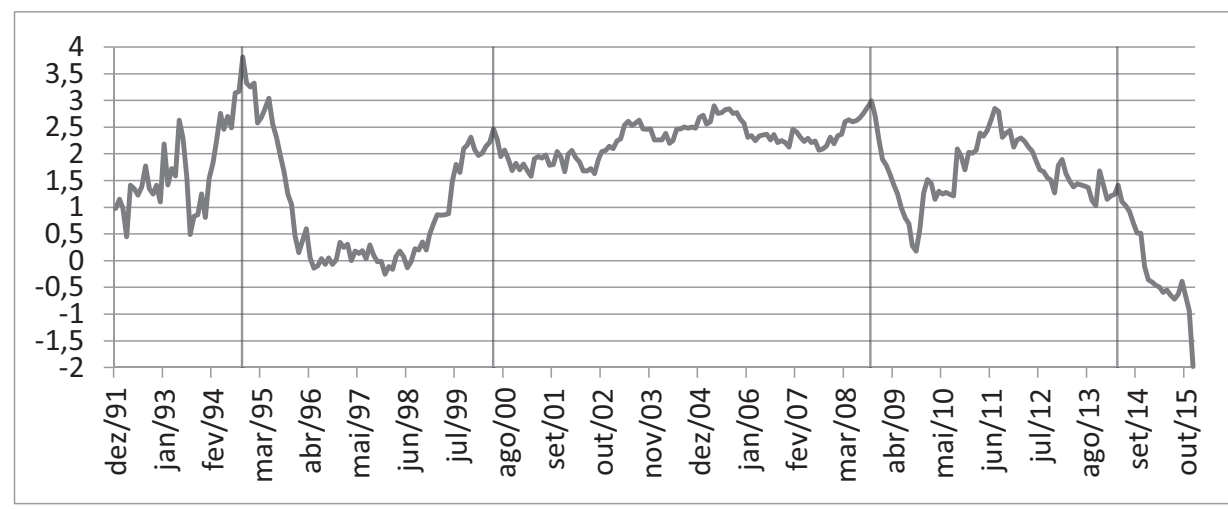

Gráfico 1 - Comportamento do resultado primário do Governo Central (\% PIB) Fonte: Banco Central.

No período de 1995 a 1998 (Primeiro Governo de Fernando Henrique Cardoso), o controle inflacionário (iniciado em 1994) reduz drasticamente os efeitos da inflação sobre os resultados fiscais do Governo. Nesse período, o resultado primário foi muito pequeno e em determinados momentos deficitário. A partir de 1999, os resultados fiscais tornam-se mais consistentes, resultantes de um grande esforço por parte do governo em cumprir o acordo firmado com o Fundo Monetário Internacional (FMI), que estabelecia metas de superávit primário.

A política fiscal adotada em resposta à crise financeira mundial, ocorrida no final de 2008, comprometeu os resultados fiscais do governo central. Os principais componentes da política fiscal brasileira em resposta à crise foram a renúncia de receita e expansão do crédito via bancos públicos. Porém, passado o epicentro da crise, o resultado primário se restabelece e volta a crescer. Em meados de 2011, contudo, observa-se uma nova inflexão do resultado primário, diferentemente do ano de 2008; a piora dos 
resultados fiscais não surge em decorrência de uma crise externa; ela é resultante de uma redefinição no direcionamento da política fiscal, em que a meta de superávit (ou a elevada austeridade fiscal) não é mais objetivo principal. A partir de setembro de 2013, os resultados entram em trajetória estritamente decrescente. $\mathrm{O}$ abandono da política de austeridade fiscal vai resultar, a partir de novembro de 2014, em seguidos déficits primários.

Os dados mostram que no final dos anos noventa o desequilíbrio era determinado pelo crescimento da receita e controle dos gastos, entretanto, a nova tendência sugere uma retração da receita acompanhada de uma elevação nos gastos. Face ao comportamento dos dados conjunturais e ao debate teórico, este trabalho se dedica a investigar qual a relação causal entre as receitas e as despesas do Governo federal brasileiro. Desta forma, este artigo tem o objetivo geral de verificar a relação de causalidade intertemporal entre receitas e gastos do governo federal brasileiro no período de 1997 a $2015^{\text {l }}$, ou seja, este artigo analisa as relações de causalidade de curto e de longo prazo entre as variáveis de receita e de gasto do governo federal. Propõem-se como objetivos específicos a aplicação de testes de raízes unitárias e de cointegração, bem como a estimação de modelos de correção de erro assimétrico (MCE-TAR e MCE-MTAR).

De forma geral, os resultados mostraram pontos relevantes: primeiro, no período de 1997 a 2013, foi possível verificar um equilíbrio de longo prazo entre a receita e a despesa do governo federal; essa estabilidade ratifica a hipótese de sustentabilidade da dívida; segundo, nesse mesmo período é identificado que a causalidade de curto prazo entre essas variáveis é do tipo tax-spend com uma relação inversa, ou seja, a relação é explicada sob a ótica da teoria da ilusão fiscal; terceiro, ao ser incorporado na análise os anos de 2014 e 2015, percebe-se que a relação de equilíbrio encontrada anteriormente deixa de existir, não sendo mais possível identificar uma ordem de causalidade entre essas variáveis. Por fim, o detalhamento dos dados evidencia que os desequilíbrios recentes estão diretamente associados a uma forte redução da receita do governo federal; isso significa que o ajuste fiscal deve priorizar a elevação de receita. Uma política de maior rigidez e controle no crescimento do gasto não será suficiente para retomar a relação de equilíbrio de longo prazo verificada no período anterior.

1 Ressaltamos que a análise da relação causal por meio do MCE - TAR entre despesa e receita será realizada para os dados de 1997 a 2013. Isso se justifica pelas mudanças estruturais ocorridas no comportamento das séries que foram identificadas no exercício metodológico. 
Essa não é uma discussão inédita para a economia brasileira, no entanto, este estudo se diferencia da literatura aplicada a essa economia, como pode ser visto na seção 3, em quatro pontos principais: a) analisar a interação entre receitas e gastos do governo federal para o período que incorpora o Governo Dilma; b) testar a hipótese de cointegração linear e não linear; c) estimação de modelos de correção de erro assimétrico (MCE-TAR e MCE-MTAR); d) simular os efeitos de choques da receita e da despesa governamental.

Além desta introdução o artigo está organizado em mais 5 seções. A seção 2 apresenta uma revisão da literatura empírica. Na seção 3 é descrito um modelo teórico de formalização da relação entre receitas e gastos públicos. $\mathrm{Na}$ seção 4 são descritos os procedimentos metodológicos. Na seção 5 é realizada a discussão dos resultados. Por fim a seção 6 traz as considerações finais do artigo.

\section{Análise da literatura}

Nesta seção serão apresentados os principais estudos aplicados às economias estrangeira e brasileira. Ressalta-se que Payne (2003) faz uma revisão dos trabalhos que trazem evidências empíricas da relação intertemporal entre receitas e despesas para várias economias. Para o período de 1980 a 2002, Payne (2003) lista, aproximadamente, 46 trabalhos empíricos de séries temporais aplicados à análise da relação intertemporal de geração de receitas e despesas do governo central. A conclusão do autor foi que os resultados podem variar de acordo com o método e com o período de tempo analisado, mas na maioria dos trabalhos aplicados, a hipótese tax-spend é a que apresenta maior suporte empírico. Além disso, Payne (2003) destaca que o método de análise mais utilizado nesses trabalhos foi o teste de causalidade Granger entre receitas e despesas. A partir do final dos anos 80 , com a introdução do modelo de cointegração desenvolvido por Engle e Granger (1987), alguns trabalhos passaram a utilizar o vetor de correlação de erros bivariados. A intuição obtida a partir dessa análise é que se as séries de receita e despesas forem cointegradas, então o modelo de correção de erros permite analisar a dinâmica de curto e longo prazo existente na relação entre as variáveis. 
Partindo dessa discussão, Romer e Romer (2009) testaram a hipótese proposta por Friedman (1978) para os EUA, na qual reduções de impostos reduzem gastos, hipótese também conhecida na literatura como starve the beast. A análise foi baseada em uma narrativa das principais mudanças ocorridas na legislação fiscal americana no período de 1945 a 2007. Os autores verificaram que não há evidências que comprovem a validade da hipótese starve the beast a economia americana. Os resultados encontrados por Romer e Romer (2009) sugerem que corte nos impostos tende a aumentar os gastos, dando suporte para a teoria de ilusão fiscal. Essa hipótese também foi testada para a economia americana no trabalho de Young (2009). Os resultados foram semelhantes aos encontrados por Romer e Romer (2009). Entretanto, Young (2009) estimou um modelo no qual o governo está sujeito a uma restrição orçamentária intertemporal, indicando que há um limite para as receitas e despesas do governo. Os resultados obtidos por esse autor também reforçam a presença de ilusão fiscal na economia dos EUA. Segundo Young (2009), a hipótese starve the beast tem sido algo já consolidado nos trabalhos sobre a economia americana, e encontrar sustentação para a hipótese de ilusão fiscal é uma reconsideração importante para subsidiar o debate sobre política fiscal.

Para Saunoris e Payne (2010), o fato de os países desenvolvidos terem repetidos déficits orçamentários nas últimas décadas, ficando mais frequente no período que sucede a crise Subprime, ampliou o interesse de gestores e pesquisadores em entender as relações causais entre receita e despesa do governo central. Nesse sentido, Saunoris e Payne (2010) analisaram as relações entre receitas e gastos do governo do Reino Unido no período de 1955 a 2009, e estimaram um modelo de correção de erro assimétrico (TAR e MTAR). Os resultados dão suporte à hipótese spend-tax com ajuste assimétrico, em que respostas do governo são mais rápidas quando há uma piora no orçamento. Seguindo a mesma direção desse estudo, Paleologou (2013) analisou as relações entre receita e despesa para a Suécia, Alemanha e Grécia. Os procedimentos metodológicos adotados foram os mesmos propostos por Payne (2010). Os resultados mostraram que, para a Suécia e a Alemanha, os choques são simétricos, a relação causal entre receita e despesa é bidirecional, reforçando a hipótese de sincronismo fiscal. No entanto, a Grécia caracterizou-se pelo fato de os choques serem assimétricos e a relação causal ser do tipo spend-tax. 
Essa ambiguidade de resultados, seja para o mesmo país ou para países distintos, reforça o argumento de que não há na literatura um consenso sobre as relações entre receitas e gastos governamentais. Para Romer e Romer (2009), esta é uma questão claramente empírica, e não encontra sustentação para uma hipótese, não implica refutá-la definitivamente. Por outro lado, Owoye e Onafowora (2011) realizaram um estudo para 22 países membros da OCDE, e encontraram resultados que dão suporte (em países distintos) às hipóteses de spend-tax, starve the beast, sincronismo fiscal e independência institucional (ausência de causalidade entre receita e despesa).

Passada a etapa de discussão de estudos aplicados a literatura estrangeira, é importante ressaltar a forma como essa relação vem sendo tratada pela literatura brasileira. De modo geral, os estudos direcionados a essa economia possuem como característica principal a análise de sustentabilidade da dívida pública e/ou no exame de choques fiscais. Dessa forma, a descrição da relação intertemporal entre receitas e despesas surge como um subproduto do resultado principal dos trabalhos. Esta característica resulta em um menor detalhamento na análise da relação entre receitas e despesas. Outro ponto comum é o método de análise e a forte interseção entre os dados dos diversos trabalhos. Os resultados, em sua grande maioria, apontam para uma relação do tipo spend-tax. Entretanto, os estudos não relatam se a interpretação deste resultado se dá via modelo de equivalência ricardiana, desenvolvido por Barros $(1974,1979)$, ou pela visão da teoria de ilusão fiscal.

Um dos primeiros estudos aplicados a economia brasileira, voltado para essa temática, foi o proposto por Mattos e Rocha (2001), que examinaram o equilíbrio orçamentário brasileiro no período de 1965 a 1993, focando as relações de curto prazo entre os componentes do orçamento fiscal do governo federal. Os autores sugeriram que, para o período analisado, a correção monetária que indexava a dívida funcionava como importante mecanismo de correção dos desvios de curto prazo do orçamento, ou seja, a redução não anunciada da correção monetária da dívida desempenhava o papel de uma elevação dos impostos. Também foi possível verificar que, no curto prazo, as relações entre gastos e receitas do governo federal foram do tipo spend-tax, sugerindo que uma elevação na arrecadação se dava em reação ao aumento nos gastos. 
Posteriormente, Mello (2005) faz uma análise do ajuste fiscal brasileiro no período que sucede a implementação do plano real (1995 a 2004), estimando uma função de reação fiscal para os gastos do governo federal e para os gastos consolidados das três esferas de governo. Os resultados indicaram que o superávit primário se eleva com a variação positiva da dívida, e que variáveis institucionais (objetivamente a Lei de Responsabilidade Fiscal - LRF) também possuem efeito positivo sobre o superávit primário. $\mathrm{Na}$ análise da sustentabilidade da dívida do governo federal (reduziu-se a amostra para o período de 1998 a 2004), os resultados demonstraram que as séries da receita primária e do gasto primário do governo federal são cointegradas, sugerindo uma dinâmica sustentável no longo prazo, cujas receitas são fortemente afetadas pelo gasto e o inverso não é válido. Desta forma, os achados do trabalho de Mello (2005) sugerem que a relação causal entre receita primária e despesa primária do governo federal é do tipo spend-tax. Esse resultado também foi obtido por Bicalho (2005), Silva et al. (2010) e Bertussi e Triches (2012), sugerindo que uma elevação no gasto pode gerar um aumento muito maior na arrecadação futura.

Diferentemente dos estudos apresentados anteriormente, Peres e Ellery (2009) estimaram os efeitos dos choques fiscais sobre a trajetória do PIB brasileiro no período de 1994 a 2005. A conclusão proposta pelos autores foi que os choques fiscais possuem características keynesianas na economia brasileira, gerando resultados positivos para elevação do gasto e efeitos negativos para elevação de impostos. Com relação à dinâmica do ajuste fiscal de curto prazo, os resultados sugerem duas direções: controle dos gastos e elevação de impostos. Desta forma, para o período de análise há evidências para uma relação bicausal entre gastos e receitas, implicando a aceitação da hipótese teórica de sincronismo fiscal. Gadelha e Divino (2013) estudaram o comportamento cíclico da política fiscal no Brasil para o período de 2002 a 2011. Eles verificaram efeitos positivos da variabilidade tributária sobre o comportamento pró-cíclico da política fiscal. Ou seja, evidenciam uma relação causal entre receita e despesa do tipo tax-spend, definindo uma relação direta entre receitas e gastos.

Por fim, mas não menos importante, ressalta-se o estudo proposto por Gadelha (2011), que verificou a relação de causalidade intertemporal entre a receita primária e o gasto total do governo federal, usando dados do período de 1997 a 2009. Na análise de causalidade foram incorporadas as variáveis PIB e Dívida. Os resultados da análise de causalidade multivariada e bivariada evidenciaram uma relação de sincronismo fiscal entre recei- 
tas e despesas. Entretanto, a relação de causalidade de longo prazo, dada pelo coeficiente de cointegração, não permite um resultado conclusivo. $\mathrm{Na}$ análise multivariada fica evidenciado o sincronismo fiscal, por outro lado, a análise bivariada corrobora com a hipótese do tipo spend-tax.

O período analisado por Gadelha (2011) possui uma forte interseção com o período de análise proposto neste artigo. Entretanto, o período de análise se estende até o ano de 2015, sendo incluído na discussão uma metodologia que permite verificar os efeitos assimétricos no processo de ajuste dos gastos e das receitas do governo federal. ${ }^{2}$ Outra diferença importante está relacionada às variáveis utilizadas. Gadelha (2011) usou as receitas primárias e os gastos totais deflacionados. Neste artigo serão utilizadas as séries de receita total e despesa total em razão do PIB. ${ }^{3}$

\section{Modelo teórico}

A relação causal entre receita e despesa pode ser expressa no modelo que analisa se o governo respeita ou não a sua restrição orçamentária intertemporal. Hakkio e Rush (1991) sugerem que se a receita governamental e despesa governamental são cointegradas, a condição de restrição orçamentária é respeitada. Este resultado é comumente utilizado como evidência de sustentabilidade da dívida pública.

Hakkio e Rush (1991) assumem uma restrição orçamentária de um período dada por:

$$
G_{t}+\left(1+i_{t}\right) B_{t-1}=R_{t}+B_{t}
$$

sendo $G_{t}$ os gastos governamentais com consumo de bens e serviços e pagamentos de transferências (não inclui pagamento de juros sobre a dívida); $B_{t}$ é a dívida pública; $R_{t}$ é a receita do governo e; $i_{t}$ é a taxa de juros real.

2 Segundo Paleologou (2013), o reconhecimento da assimetria permite uma melhor compreensão dos efeitos dos déficits e superávits sobre a dinâmica da receita e do gasto. Além disso, a autora argumenta que se o ajuste é assimétrico a suposição de simetria leva a uma má especificação do modelo estimado.

3 Segundo Hondroyiannis e Papapetrou (1996), o uso das variáveis em razão do PIB justifica-se pela redução da dependência em relação à dinâmica nominal da renda e porque os principais indicadores fiscais, tais como dívida e déficit, são sempre utilizados em razão do PIB. 
Resolvendo a Equação (1) para cada unidade de tempo no futuro tem-se:

$$
B_{0}=\sum_{t-1}^{\infty} r_{t}\left(R_{t}-G_{t}\right)+\lim _{n \rightarrow \infty} r_{n} B_{n}, r_{t}=\prod_{s=1}^{t}\left(1+i_{s}\right)^{-1}
$$

A condição de sustentabilidade exige que a dívida atual seja financiada por excedentes de receitas futuros. Esta condição implica:

$$
\lim _{n \rightarrow \infty} r_{n} B_{n}=0
$$

Caso essa condição não seja respeitada, então a dívida estará sendo financiada pela criação de novas dívidas, fato que se caracteriza por ser um sistema de financiamento do tipo Ponzi. A sustentabilidade da dívida está associada à ideia de que, no limite do prazo, a dívida será zero. Desta forma, é esperado que a taxa de crescimento médio do estoque de dívida não seja maior do que a taxa de crescimento médio da economia.

Baseado nessa hipótese, Hakkio e Rush (1991) assumem que, se a taxa de juros da economia for estacionária, então é possível transformar a Equação (1) na seguinte relação de longo prazo entre receita e despesa governamental:

$$
R_{t}=\gamma_{o}+\gamma_{1} \tilde{G}_{t}+\varepsilon_{t}
$$

Em que $\widetilde{G}_{t}$ é o gasto governamental incluindo o pagamento de juros sobre a dívida; $\gamma_{o}$ e $\gamma_{1}$ são os parâmetros de cointegração e; $\varepsilon_{t}$ é o resíduo e esse reflete o desequilíbrio entre receita e despesa governamental. A hipótese de sustentabilidade da dívida assume que $\gamma_{1}=1$ e $\varepsilon_{t}$ possui um comportamento estacionário.

\section{Abordagem metodológica}

Para alcançar os objetivos propostos pelo estudo serão adotadas duas abordagens metodológicas principais. A primeira abordagem é voltada para a análise de cointegração entre as séries de receita e gasto do governo, sendo 
utilizado os testes de cointegração de Engle e Granger, Johansen e PhillipsOuliaris para captar essa relação. Ressalta-se que uma condição necessária para o uso dos testes de cointegração é a verificação da estacionariedade das séries, assim, inicialmente serão aplicados os testes de raiz unitária de Dickey-Fuller aumentado e KPSS, com o intuito de identificar a ordem de integração das séries e confrontar os resultados obtidos por meio de testes que possuem hipóteses de identificação de raiz unitária opostas.

Um ponto relevante e que deve ser levado em consideração nessa análise é a possibilidade de ocorrência de mudanças estruturais nas séries. Assim, de forma complementar, será aplicado o teste de raiz unitária com quebra estrutural proposto por Lee-Strazicich. Dessa forma, caso seja identificada uma mudança estrutural nas séries, é preciso realizar uma análise adicional por meio de testes de cointegração que levem em consideração esse componente. Isso porque todos os testes de cointegração que serão adotados na primeira parte dessa discussão perdem validade, além de não levarem em consideração a possibilidade de as séries manterem uma relação de cointegração não linear. Será adotado o teste de cointegração proposto por Gregory e Hansen (1996) para verificar se há uma mudança estrutural na relação de cointegração das séries.

Caso as séries de receita e despesa do governo federal mantenham uma relação de equilíbrio de longo prazo, então essa relação será modelada por meio do modelo de correção de erro. Esse modelo tem as características de um vetor autorregressivo (VAR) ${ }^{4}$ incorporando o erro de equilíbrio; por isso, denomina-se vetor de correção de erros (VEC). Esse modelo pode ser representado pelo seguinte sistema de equações:

$$
\begin{aligned}
& \Delta R_{t}=\mathrm{A}_{0}+\delta_{1} \hat{\varepsilon}_{t-1}+\sum_{j=1}^{k-1} \alpha_{11, j} \Delta R_{t-j}+\sum_{j=1}^{k-1} \beta_{11, j} \Delta D_{t-j}+\mu_{y t} \\
& \Delta D_{t}=\mathrm{B}_{0}+\delta_{2} \hat{\varepsilon}_{t-1}+\sum_{j=1}^{k-1} \alpha_{21, j} \Delta R_{t-j}+\sum_{j=1}^{k-1} \beta_{21, j} \Delta D_{t-j}+\mu_{z t}
\end{aligned}
$$

\footnotetext{
4 Ver Johansen (1995) e Engle e Granger (1987).
} 
As Equações 5 e 6 permitem analisar as relações de longo prazo, representadas pelos coeficientes $\delta_{1}$ e $\delta_{2}$, bem como as relações de curto prazo capturadas pelos coeficientes $\alpha_{j}$ e $\beta_{j}$. Os coeficientes $\delta_{1}$ e $\delta_{2}$ medem a velocidade do ajuste ao equilíbrio de longo prazo. A partir dessas equações é possível observar as relações de causalidade no sentido de Granger ${ }^{5}$ entre as variáveis de interesse. Segundo Granger (1988), a cointegração entre duas variáveis sugere pelo menos uma relação de causalidade unidirecional. As relações de causalidade podem ser verificadas pela significância individual dos coeficientes de longo prazo $\left(\delta_{1}\right.$ e $\left.\delta_{2}\right)$ e pela significância conjunta dos coeficientes da relação de curto prazo $\left(\alpha_{j}\right.$ e $\left.\beta_{j}\right)$.

Em discussão mais recente, Enders e Siklos (2001) propuseram uma análise de cointegração a qual permite que o termo correção seja assimétrico. Ou seja, a velocidade de ajuste ao equilíbrio longo prazo é assimétrico; assim, desvios positivos e negativos terão respostas diferentes. Os autores sugerem investigar a cointegração com a inclusão de um limiar (threshold) com duas possíveis formalizações: threshold autoregressive (TAR) e momentum threshold autoregressive cointegration (MTAR).

A verificação de cointegração com threshold autoregressive (TAR) pode ser expressa por:

$$
\Delta \widehat{\varepsilon_{t}}=\rho_{1} I_{t} \hat{\varepsilon}_{t-1}+\rho_{2}\left(1-I_{t}\right) \hat{\varepsilon}_{t-1}+\sum_{i=1}^{k-1} \lambda_{i+t} \Delta \hat{\varepsilon}_{t-1}+v_{t}
$$

Em que, $I_{t}$ é uma variável dummy, tal que:

$$
I_{t}= \begin{cases}1, & \text { se } \hat{\varepsilon}_{t-1} \geq \tau \\ 0, & \text { se } \quad \hat{\varepsilon}_{t-1}<\tau\end{cases}
$$

O parâmetro $\tau$ é threshold que divide a série. Este parâmetro pode ser estimado ou, como descrito na literatura, pode ser estabelecida uma restrição na qual esse parâmetro passa a assumir valor zero. No caso do modelo MTAR, a variável $I_{t}$ assume os seguintes valores:

$$
I_{t}= \begin{cases}1, & \text { se } \Delta \hat{\varepsilon}_{t-1} \geq \tau \\ 0, & \text { se } \Delta \hat{\varepsilon}_{t-1}<\tau\end{cases}
$$

5 A ideia de causalidade expressa em Granger (1969) sugere uma precedência temporal entre as variáveis de forma a realizar melhores previsões. 
A representação do MTAR sugere que a série possui maior dinâmica em uma direção do que em outra. Em ambas as especificações (TAR e MTAR), a condição suficiente para estacionariedade do resíduo estimado $\left(\widehat{\varepsilon_{t}}\right)$ é dada por $-2<\left(\rho_{1}, \rho_{2}\right)<0$. A hipótese nula de não cointegração é testada pelo F-estatístico da hipótese nula de $\rho_{1}=\rho_{2}=0 .{ }^{6}$ Se a hipótese nula de não cointegração for rejeitada, verificamos a simetria que é testada pelo F-estatístico em que a hipótese nula é dada por $\rho_{1}=\rho_{2}$.

As Equações 7, 8 e 9 são consistentes com o modelo de correção de erros. Podemos escrever o modelo de correção de erro com threshold no seguinte sistema de equações:

$$
\begin{aligned}
& \Delta R_{t}=\mathrm{A}_{0}+\rho_{1} I_{t} \hat{\varepsilon}_{t-1}+\rho_{2}\left(I_{t}-1\right) \hat{\varepsilon}_{t-1}+\sum_{j=1}^{k-1} \alpha_{11, j} \Delta R_{t-j}+\sum_{j=1}^{k-1} \beta_{11, j} \Delta D_{t-j}+\mu_{y t} \\
& \Delta D_{t}=\mathrm{A}_{0}+\tilde{p}_{1} I_{t} \hat{\varepsilon}_{t-1}+\tilde{\rho}_{2}\left(I_{t}-1\right) \hat{\varepsilon}_{t-1}+\sum_{j=1}^{k-1} \alpha_{11, j} \Delta R_{t-j}+\sum_{j=1}^{k-1} \beta_{11, j} \Delta D_{t-j}+\mu_{y t}
\end{aligned}
$$

As Equações 10 e 11 permitem realizar as mesmas análises das relações de longo e curto prazo do modelo de correção de erro simétrico das Equações 5 e 6. Entretanto, a existência de assimetria no ajuste de longo prazo implica em erro de especificação do modelo simétrico. Ademais, do ponto de vista de análise, os ajustes assimétricos permitem uma melhor compreensão das relações de equilíbrio entre as variáveis econômicas.

Por fim, serão simulados choques de receita e gasto do governo, por meio do modelo VAR, como análise de robustez. Essa análise tem como finalidade principal verificar se as conclusões obtidas na primeira parte do artigo continuam válidas quando alterado o método de análise.

6 O teste não segue a distribuição padrão. Os valores críticos estão disponíveis em Enders e Siklos (2001). 


\subsection{Descrição dos dados}

Os dados utilizados nesta pesquisa são referentes ao período de janeiro de 1997 a dezembro de 2015. Os dados são mensais acumulados dos últimos doze meses. As fontes de dados são a Secretaria do Tesouro Nacional (STN) e o Instituto de Economia Aplicada (IPEA). O Quadro 1 apresenta a descrição de variáveis utilizadas.

Quadro 1 - Descrição das variáveis

\begin{tabular}{|l|l|}
\hline \multicolumn{1}{|c|}{ Variáveis } & \multicolumn{1}{c|}{ Descrição } \\
\hline Receita Total & $\begin{array}{l}\text { Dada pela soma da receita do tesouro nacional (arrecadação de impostos, contribuições e } \\
\text { outras receitas), da receita da previdência, receita do Banco do Brasil e das transferências } \\
\text { para estados e municípios. }\end{array}$ \\
Despesa Total & $\begin{array}{l}\text { Dada pelo soma das despesas com pessoal e encargos sociais, benefícios previdenciários, } \\
\text { custeio e capital, transferências do Tesouro ao Banco Central e despesas do Banco Central. }\end{array}$ \\
PIB & $\begin{array}{l}\text { Produto Interno Bruto (PIB) acumulado dos últimos 12 meses (Valores correntes). Estima- } \\
\text { tiva do Banco Central. }\end{array}$ \\
\hline
\end{tabular}

Fonte: Elaboração própria.

\section{Resultados e discussões}

No Gráfico 2 são apresentadas as séries de receita e despesa do governo central brasileiro, em proporção da estimativa mensal do PIB, no período de dezembro de 1997 a dezembro de 2015. É possível observar que as contas públicas apresentaram diferenças marcantes entre o período que antecedeu o ano de 2013 e o período que sucedeu, sendo o segundo período caracterizado pelos desequilíbrios fiscais e pelo aumento da vulnerabilidade das finanças do governo central. Como pode ser visto, a dinâmica da receita e despesa do governo central segue um comportamento muito parecido até o ano de 2013, com receita sendo superior a despesa. A partir do ano seguinte a receita começa a cair, sendo acompanhada de uma aparente expansão das despesas em proporção do PIB. 


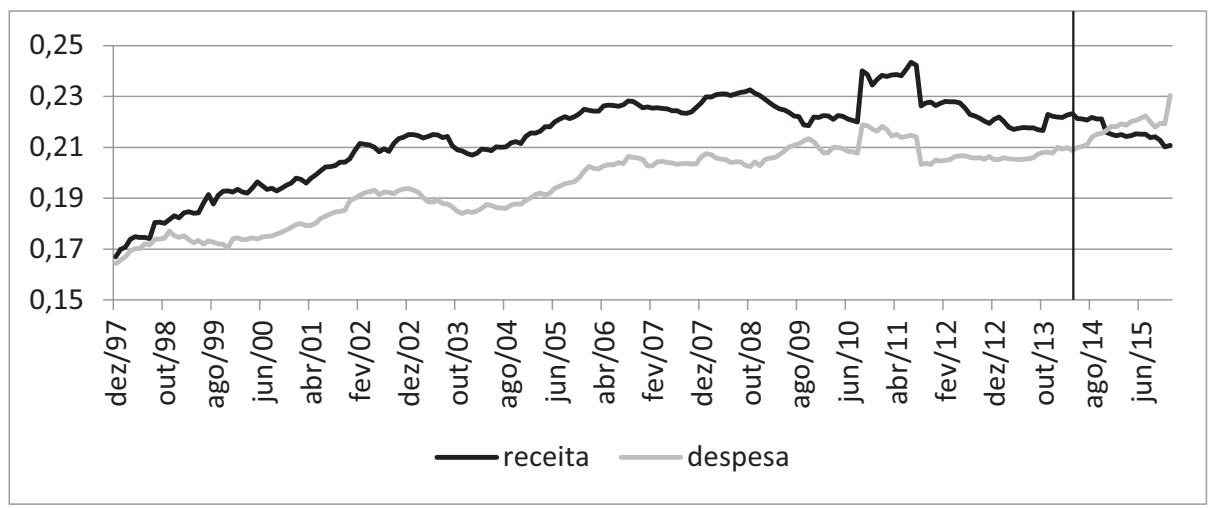

Gráfico 2 - Receita e despesa do governo central brasileiro, em proporção do PIB, no período de dezembro de 1997 a dezembro de 2015

Fonte: Elaboração própria.

Diante dessa mudança de comportamento, surge a primeira indagação: o que ocorreu nesse período para haver uma alteração no comportamento dessas séries? A primeira explicação que pode surgir na mente é a relacionada ao ciclo político, sendo uma característica muito comum para anos eleitorais. No entanto, por mais que esse componente possa estar presente, não é possível concluir que esse foi o único fator responsável pela mudança na trajetória da receita e da despesa do governo central no ano de 2014. Talvez a explicação mais plausível para esse comportamento seja a evolução do crescimento médio do PIB real brasileiro durante esse período. Como descrito na Tabela 1, percebe-se que o PIB real apresentou um crescimento médio anual de 3,2\% no período de 1997 a 2013, passando a ter um fraco desempenho a partir de 2014, com decréscimo médio de $-1.87 \%$ para esse período.

Tabela 1 - Crescimento médio do PIB real, crescimento da receita e da despesa em proporção do $\mathrm{PIB}$, razão entre o crescimento da despesa e receita do governo central

\begin{tabular}{c|cccc}
\hline & $\Delta P I B_{t} \%$ & $\left(\Delta R_{t} / \Delta P I B_{t}\right) \%$ & $\left(\Delta D_{t} / \Delta P I B_{t}\right) \%$ & $\left(\Delta D_{t} / \Delta R_{t}\right) \%$ \\
\hline $1997 \mathrm{a} 2013$ & 3.20 & 140.22 & 132.07 & 94.19 \\
$2014 \mathrm{a} 2015$ & -1.87 & 48.31 & 200.60 & 415.26 \\
\hline
\end{tabular}

Fonte: Elaboração própria. 
A partir da Tabela 1 é possível observar que a principal mudança fiscal ocorrida na economia brasileira no período recente foi a queda na arrecadação. Percebe-se que no período de 1997 a 2013, a receita apresentava uma trajetória de crescimento superior as despesas, sendo que, a partir de 2014, com o desaquecimento da economia agregada, houve redução na arrecadação, e esta passou a crescer menos que as despesas, sinalizando os primeiros alertas. Esse cenário simplista mostra que é difícil argumentar que o problema fiscal brasileiro no período atual está ligado unicamente ao aumento das despesas, pois mesmo que a despesa nominal não aumentasse, o indicador despesa em razão do PIB iria crescer devido à redução do PIB. Além disso, é natural que haja uma redução na arrecadação em períodos de instabilidade, mas não nas proporções e na velocidade que isso tem ocorrido na economia brasileira.

É importante ressaltar que essa piora nas contas públicas da economia brasileira tem características contrárias a crise fiscal observada nas economias avançadas no período que sucedeu a crise Subprime. Países como Estados Unidos, Japão, Reino Unido e Alemanha, praticamente, não tiveram alterações na receita do governo geral no período que sucedeu essa crise. Por outro lado, houve um aumento significativo nas despesas do governo central desses países. Em 2006, por exemplo, a despesa do governo geral do Japão, Reino Unido e Estados Unidos representava 34.5\%, 40.6\% e $35.9 \%$ do PIB, passando para 40.7\%, 47.3\% e 44\%, respectivamente, em 2009. A explicação para a elevação desse indicador nessas economias está relacionada ao baixo crescimento do PIB a partir de 2008, sobretudo, devido ao aumento das medidas de apoio ao setor financeiro, envolvendo a transferência de ativos / passivos para o balanço do setor público.

Todos esses novos componentes que surgiram no período recente levam a questionamentos sobre a sustentabilidade da dívida pública brasileira, perspectivas futuras do orçamento fiscal, dentre outros. Sendo que as próximas etapas desse artigo estão voltadas para a análise da relação intertemporal entre as variáveis de despesa e receita do governo central brasileiro, com o intuito de verificar se a causalidade é unidirecional (do tipo tax-spend ou spend-tax) ou bidirecional. 


\subsection{Análise de cointegração}

Parte dos questionamentos propostos anteriormente serão respondidos a partir da análise de cointegração. Apesar de ser um método bastante discutido na literatura, é importante ressaltar que o uso desse método condiciona a aplicação de uma série de testes como condições necessárias, dentre esses, destaca-se: testes de raiz unitária e quebra estrutural. Assim, a discussão subsequente é voltada para a análise de estabilidade das séries, por meio dos testes de raiz unitária (Dickey-Fuller e KPSS).

Tabela 2 - Testes de raiz unitária

\begin{tabular}{|c|c|c|c|c|c|c|}
\hline & \multicolumn{3}{|c|}{ Dickey-Fuller } & \multicolumn{3}{|c|}{ KPSS } \\
\hline & $t_{2013}$ & $t_{2015}$ & $t_{\text {crítico }}$ & $\hat{\eta}_{2013}$ & $\hat{\eta}_{2015}$ & $\eta_{\mu}$ \\
\hline Receita & -2.99 & -3.17 & -2.87 & 0.41 & 0.52 & 0.146 \\
\hline Despesa & -2.06 & -0.87 & -2.87 & 0.27 & 0.28 & 0.146 \\
\hline$\Delta$ Receita & -3.50 & -4.40 & -2.87 & 0.03 & 0.02 & 0.146 \\
\hline$\Delta$ Despesa & -3.59 & -3.77 & -2.87 & 0.03 & 0.03 & 0.146 \\
\hline
\end{tabular}

Fonte: Elaboração própria.

Os resultados dos testes de raiz unitária de Dickey-Fuller e KPSS, apresentados na Tabela 2, sugerem que as séries de receita e da despesa do governo central apresentam tendência estocástica em nível, passando a ser estacionária em primeira diferença $\mathrm{I}(1)$. Esses resultados reforçam o argumento que as séries de receita e despesa do governo central possuem comportamento explosivo em nível, ficando difícil projetar ou tirar qual conclusão acerca do comportamento futuro dessas variáveis. Assim, qualquer análise derivada do uso dessas séries deve levar em consideração esse componente. Nesse artigo o tratamento dado foi a retirada da componente tendência de ambas variáveis, tornando-as séries estáveis.

Passada essa etapa, a discussão seguinte é voltada para a análise dos testes de cointegração entre as séries de receita e despesa do governo central. Com o intuito de confrontar os resultados obtidos a partir de diferentes testes, foi realizada uma análise comparativa entre os testes de cointegração de Engle-Granger, Johansen e Phillips-Ouliaris, tal como apresentado na Tabela 3. 
Tabela 3 - Testes de cointegração propostos por Johansen, Engle-Granger e PhillipsOuliaris

\begin{tabular}{|c|c|c|c|c|c|c|c|c|}
\hline & \multicolumn{4}{|c|}{ Johansen } & \multicolumn{2}{|c|}{ Engle-Granger } & \multicolumn{2}{|c|}{ Phillips-Ouliaris } \\
\hline Período & & $\begin{array}{l}\text { Autovalor } \\
\text { máximo }\end{array}$ & Traço & $\begin{array}{l}\text { Traço } \\
(95 \%)\end{array}$ & & & & \\
\hline \multirow[b]{2}{*}{$97-13$} & 0 & 17.12 & 33.24 & 15.41 & Est. teste & -4.86 & Est. teste & -3.90 \\
\hline & 1 & 16.12 & 16.12 & 3.84 & $\begin{array}{l}\text { Valor } \\
\text { crítico }\end{array}$ & -3.37 & $\begin{array}{l}\text { Valor } \\
\text { crítico }\end{array}$ & -3.36 \\
\hline \multirow[b]{2}{*}{$97-15$} & 0 & 18.67 & 30.69 & 15.41 & Est. teste & -3.80 & Est. teste & -4.01 \\
\hline & 1 & 12.02 & 12.02 & 3.84 & $\begin{array}{l}\text { Valor } \\
\text { crítico }\end{array}$ & -3.36 & $\begin{array}{l}\text { Valor } \\
\text { crítico }\end{array}$ & -3.36 \\
\hline
\end{tabular}

Fonte: Elaboração própria.

Todos os testes apresentados na Tabela 3 mostraram que as séries de receitas e despesas do governo central são cointegradas, dando fortes indícios que a dívida pública brasileira é sustentável e que o governo respeitou sua restrição orçamentária no período de análise. No entanto, é importante ressaltar que os testes de cointegração utilizados acima perdem validade na presença de uma mudança estrutural nas séries ou na relação de cointegração dessas. Essa discussão está bem documentada no estudo de Bierens (1997), cujos testes convencionais de cointegração mostram não conseguir captar a verdadeira natureza do processo de ajustamento das séries quando esse processo é dado de forma não linear ou quando há mudança de regime. Assim, para contornar essa possível limitação, foi aplicado o teste de raiz unitária com quebra estrutural nas séries de receita e despesa, proposto por Lee-Strazicich e, na sequência, foi aplicado o teste de cointegração com quebra estrutural sugerido por Gregory e Hansen (1996).

Na Tabela 4 são apresentados os resultados obtidos a partir do teste de quebra estrutural com a inclusão de mudança no nível da série, denominado de Modelo A, e do teste que capta possíveis quebras estruturais no nível e na tendência da série, também chamado de Modelo C. Os testes reforçam os resultados já obtidos pelos testes de DickeyFuller e KPSS, onde as séries foram caracterizadas pela presença de raiz unitária, porém inclui um novo componente na análise que é a presença de uma quebra estrutural nas séries. 
Tabela 4 - Teste de raiz unitária com quebra proposto por Lee-Strazicich

\begin{tabular}{|c|c|c|c|c|c|c|c|c|}
\hline & \multicolumn{3}{|c|}{ Modelo A } & \multirow[b]{2}{*}{ Quebra } & \multicolumn{4}{|c|}{ Modelo C } \\
\hline & $t_{97-13}$ & Quebra & $t_{97-15}$ & & $t_{97-13}$ & Quebra & $t_{97-15}$ & Quebra \\
\hline Receita & -2.76 & 2007:06 & -3.15 & 2007:09 & -1.79 & 2011:08 & -1.71 & 2011:08 \\
\hline Despesa & -3.31 & 2011:05 & -3.58 & 2011:07 & -2.66 & 2011:08 & -1.88 & 2011:08 \\
\hline
\end{tabular}

Fonte: Elaboração própria.

Esse achado é bastante curioso, tendo em vista que outros autores, tais como, Pastore, Gazzano e Pinotti (2014) e Nóbrega (2016), também ressaltaram mudanças políticas e estruturais para esse período. Pastore, Gazzano e Pinotti (2014) mencionaram que o ano de 2011 foi marcado pela mudança de regime na política econômica que, na prática, consistiu do abandono do tripé macroeconômico. Assim, o passo adiante está relacionado à inclusão da quebra estrutural nos testes de cointegração. Essa discussão, como já ressaltado, será realizada a partir do teste de cointegração de Gregory e Hansen (1996).

Tabela 5 - Teste de cointegração de Gregory e Hansen (1996)

\begin{tabular}{c|cccc}
\hline Período & $t_{\text {stat }}$ & Valor crítico & Quebra & Resultado \\
\hline $1997-2013$ & -5.042 & -4.950 & $2011: 02$ & Cointegração \\
$1997-2015$ & -5.262 & -5.500 & $2012: 11$ & Não cointegração \\
\hline
\end{tabular}

Fonte: Elaboração própria.

Nota: A hipótese nula do teste Gregory e Hansen (1996) afirma que as séries são não cointegradas.

Levando em consideração a quebra estrutural na relação de cointegração, é possível afirmar que a dívida pública brasileira foi sustentável até o ano de 2013, quando as séries de receita e despesa foram cointegradas. Por outro lado, quando são incluídos os anos de 2014 e 2015 na análise, passam a surgir os primeiros indícios de perda de controle fiscal no Brasil, fato corroborado pelo teste de cointegração de Gregory e Hansen (1996) que mostra que as séries tratadas deixam de ser cointegradas e que há uma mudança estrutural na relação entre essas em novembro de 2012. 
Toda a discussão que foi apresentada até esta parte do artigo é relevante para sugerir fatores que podem ter alterado a relação entre receita e gasto no período de análise, mas não é suficiente para responder o problema de pesquisa proposto inicialmente, em que se procura verificar a relação causal entre as variáveis de gasto e receita do governo federal brasileiro. Assim, com base na discussão anterior, surgem dois novos cenários: o primeiro é o que descreve uma situação de dívida sustentável, tal como observado para o período de 1997 a 2013; o segundo é uma situação de dívida não sustentável.

Nesse caso, só será possível captar a relação causal entre as variáveis de gasto e receita do governo federal brasileiro para a situação de dívida sustentável, tendo em vista que, quando as séries são cointegradas, é possível captar a direção que uma série vai seguir quando ocorre alteração na outra, fato não observado no caso de não cointegração, discussão que será abordada na próxima subseção.

\subsection{Modelos TAR e MTAR}

A relação causal entre esses fatores será tratada a partir da análise de cointegração com efeitos assimétricos, realizada por meio dos modelos TAR e MTAR com uma defasagem, tal como apresentado na Tabela 6. 
Tabela 6 - Estimações dos testes de cointegração não linear (TAR e MTAR) ${ }^{7}$

\begin{tabular}{|c|c|c|}
\hline & TAR & MTAR \\
\hline$\rho_{1}$ & $-0,8375^{\star \star \star}$ & $-0,6403^{\star * *}$ \\
\hline$\rho_{2}$ & $-0,4745^{\star \star \star}$ & $-0,5609^{\star \star \star}$ \\
\hline$\Lambda$ & $-0,0779$ & $-0,1077$ \\
\hline$\tau$ & 0,0041 & 0,0004 \\
\hline \multicolumn{3}{|l|}{ Testes } \\
\hline$H_{o}$ : Não Cointegração & 27,98 & 26,60 \\
\hline \multicolumn{3}{|l|}{$\rho_{1}=\rho_{2}=0$} \\
\hline Valor $\mathbf{p}$ & 0,0000 & 0,0000 \\
\hline$H_{o}:$ Simetria & 6,13 & 0,35 \\
\hline \multicolumn{3}{|l|}{$\rho_{1}=\rho_{2}$} \\
\hline Valor $\mathbf{p}$ & 0,0141 & 0,5543 \\
\hline$H_{o}:$ não correlação serial & 6,5526 & 6,8184 \\
\hline \multicolumn{3}{|l|}{$\mathrm{Q}_{\mathrm{LB}}(12)$} \\
\hline Valor $\mathbf{p}$ & 0,8857 & 0,8694 \\
\hline \multicolumn{3}{|l|}{ Estatísticas } \\
\hline Observações & 200 & 200 \\
\hline$R^{2}$ & 0,40 & 0.40 \\
\hline AIC & $-1110,952$ & $-1110,827$ \\
\hline SBC & $-1097,759$ & $-1097,634$ \\
\hline
\end{tabular}

Fonte: Elaboração própria.

Nota: $\left({ }^{*}\right),\left({ }^{* *}\right)$ e $\left({ }^{* * *}\right)$ representam o nível de significância do coeficiente, respectivamente, em $10 \%$, $5 \%$ e $1 \%$.

Ao se observar o resultado é possível perceber que a condição de estacionariedade foi respeitada e rejeitou-se a hipótese nula de não cointegração para ambas as formalizações de cointegração assimétrica (TAR e MTAR).

\footnotetext{
${ }^{7}$ O threshold $(\tau)$ foi calculado de acordo com Chan (1993).
} 
Além disso, verifica-se, com base na estatística Q de Box-Pierce, ausência de correlação nos resíduos. Adicionalmente, no modelo MTAR não foi possível rejeitar a hipótese nula de simetria, diferentemente do modelo TAR, cuja hipótese nula de simetria foi rejeitada.

Esses resultados da análise de cointegração do modelo TAR, descritos na Tabela 7, sugerem que a receita e a despesa do governo federal apresentam uma relação de equilíbrio no longo prazo, e que os ajustes são assimétricos. Assim, o governo deve apresentar respostas distintas quando há uma deterioração ou uma melhora orçamentária. Este resultado permite estimar um modelo de correção de erro assimétrico (MCE-TAR).

A Tabela 7 traz a estimação do MCE-TAR. Observa-se que os coeficientes de ajuste de longo prazo $\left(\rho_{1}\right)$, responsáveis por representar o ajuste a desvios positivos, são insignificantes do ponto de vista estatístico e, em termos absolutos, são menores do que $\rho_{2}$ (representa o ajuste a desvios negativos). Este resultado $\left(\left|\rho_{1}\right|<\left|\rho_{2}\right|\right)$ sugere que o ajuste a uma deterioração do orçamento é mais rápido do que quando ocorre uma melhora orçamentária.

Observa-se que o termo de correção de erro $\rho_{2}$ é significante nas duas equações. No caso da equação da receita, verifica-se que o termo de correção de erro é negativo, por outro lado, na equação da despesa o termo de correção de erro é positivo. Estes resultados indicam que os desequilíbrios orçamentários são corrigidos no longo prazo por movimentos simultâneos na receita e despesa. Este achado sugere um suporte empírico para a hipótese de sincronismo fiscal, uma vez que, no longo prazo, há uma causalidade bidirecional entre receita e despesa.

$\mathrm{Na}$ equação da receita os coeficientes da relação de curto prazo $\left(\Delta\right.$ Despesa $_{t-1}, \Delta$ Despesa $\left._{t-2}\right)$ não são significativos do ponto de vista estatístico. Na equação da despesa todos os coeficientes da relação de curto prazo $\left(\Delta\right.$ Receita $_{t-1}, \Delta$ Receita $_{t-2}, \Delta$ Despesa $_{t-1}$ e $\Delta$ Despes $\left._{t-2}\right)$ são significativos do ponto de vista estatístico. $\mathrm{Na}$ análise de curto prazo, o resultado do teste de causalidade Granger sugere que, no curto prazo, a despesa não causa receita, mas receita causa despesa, de forma significativa (ao nível de $5 \%$ ), na segunda defasagem. 
Tabela 7 - Estimação do Modelo de Correção de Erro assimétrico (MCE-TAR)

\begin{tabular}{|c|c|c|}
\hline Variável & $\Delta$ Receita & $\Delta$ Despesa \\
\hline$\rho_{1}$ & $-0,2700$ & 0,0522 \\
\hline$\rho_{2}$ & $-0,3710^{* * *}$ & $0,1424^{\star *}$ \\
\hline$\Delta$ Receita $_{t-1}$ & $-0,7408^{\star \star *}$ & $-0,0190$ \\
\hline$\Delta$ Receita $_{t-2}$ & $-0,3960^{\star \star \star}$ & $-0,1021^{* *}$ \\
\hline$\Delta$ Despesa $_{t-1}$ & 0,1990 & $-0,6105^{\star \star \star}$ \\
\hline$\Delta$ Despesa ${ }_{t-2}$ & 0,0468 & $-0,2692^{\star * *}$ \\
\hline Constante & $-0,0009$ & 0,0014 \\
\hline Obs. & 200 & 200 \\
\hline$R^{2}$ & 0,41 & 0,31 \\
\hline AIC & $-1133,269$ & $-1345,564$ \\
\hline SBC & $-1110,180$ & $-1322,476$ \\
\hline$Q_{L B}(12)$ & 9,3833 & 17,1748 \\
\hline \multicolumn{3}{|l|}{ Análise de Curto Prazo } \\
\hline Hipótese Nula & $\chi^{2}$ & Prob. $>\chi^{2}$ \\
\hline $\begin{array}{l}\Delta \text { Desp. não Causa Granger } \Delta \text { Rec. } \\
\qquad\left(\beta_{1}=\beta_{2}=0\right)\end{array}$ & 1,36 & 0,2585 \\
\hline $\begin{array}{l}\Delta R e c \text {. não Causa Granger } \Delta \text { Desp. } \\
\qquad\left(\alpha_{1}=\alpha_{2}=0\right)\end{array}$ & 2,52 & 0,0831 \\
\hline
\end{tabular}

Fonte: Elaboração própria.

Nota: $\left({ }^{*}\right),\left({ }^{* *}\right)$ e $\left({ }^{* * *}\right)$ representam o nível de significância do coeficiente, respectivamente, em $10 \%$, $5 \%$ e $1 \%$.

Os coeficientes da relação de curto prazo na equação da despesa indicam que a receita afeta a despesa de forma negativa, ou seja, elevações na receita devem reduzir o gasto. Este resultado implica um suporte empírico para uma relação do tipo tax-spend, em conformidade com a teoria de ilusão fiscal.

Os resultados sugerem duas possíveis interpretações para a relação intertemporal entre receita e despesas do governo federal. Na relação de longo prazo pode ser interpretado aos moldes da hipótese de sincronismo fiscal apresentada por Meltzer and Richard (1981). Entretanto, a relação de curto prazo sugere uma interpretação do tipo tax-spend aos moldes 
da teoria de ilusão fiscal. Esse achado implica uma relação unidirecional entre receita e despesa, e ao contrário da maioria dos trabalhos aplicados ao Brasil, despesa não causa receita. Em outras palavras, o resultado sugere que reduções na receita, no curto prazo, devem elevar os gastos.

Em síntese, as análises mostraram: os ajustes ao equilíbrio de longo prazo são realizados em ambas as variáveis. Ou seja, há evidência de que, no longo prazo, mudanças no comportamento da receita explicam as mudanças no comportamento da despesa e vice-versa; no longo prazo a causalidade bidirecional corrobora a teoria de sincronismo fiscal; os ajustes de longo prazo são assimétricos, cujos desvios negativos do orçamento são corrigidos de forma mais rápida; ao contrário da maior parte dos resultados encontrados para o Brasil, não há evidências para relação do tipo spend-tax; as relações de curto prazo entre receita e despesa são do tipo tax-spend, em que reduções na receita devem elevar a despesa; os resultados da análise de curto prazo estão em conformidade com a teoria de ilusão fiscal.

A direção obtida neste artigo, para o longo prazo, assemelha-se aos achados encontrados por Gadelha (2011). Ressalta-se que Gadelha (2011) obteve uma relação causal bidirecional a partir de uma análise de causalidade, no sentido de precedência temporal, conforme proposto por Granger (1969). Por outro lado, as conclusões obtidas por este estudo baseiam-se em uma análise de causalidade funcional associada aos resultados dos sinais e dos coeficientes estimados dos coeficientes da relação de longo prazo (termos de correção de erros).

\subsection{Análise de robustez}

Nesta seção será apresentada a análise de robustez dos exercícios apresentados anteriormente, sendo adotada especificação alternativa por meio do modelo de vetores autorregressivos (VAR) irrestrito, com o emprego da análise de funções impulso-resposta generalizada. Essa análise restringese às funções impulso resposta dos choques de receita e despesa governamental. É importante ressaltar que as funções impulso resposta foram obtidas por meio do modelo VAR com uma defasagem, sendo o número defasagens definido a partir dos critérios de informação AIC, SBC e HQ, com bandas de probabilidade representadas entre 0.16 e 0.84 , horizonte de tempo de 20 meses. 
Além disso, foram incluídas as dummies de nível e inclinação como variáveis exógenas, com intuito de captar os efeitos da mudança estrutural já descritos anteriormente. Logo após a estimação do modelo foram realizados alguns testes de checagem do mesmo. Nesta etapa foi possível verificar que o modelo VAR estimado exibiu normalidade nos resíduos e não apresentou problemas de autocorrelação residual e heterocedasticidade.
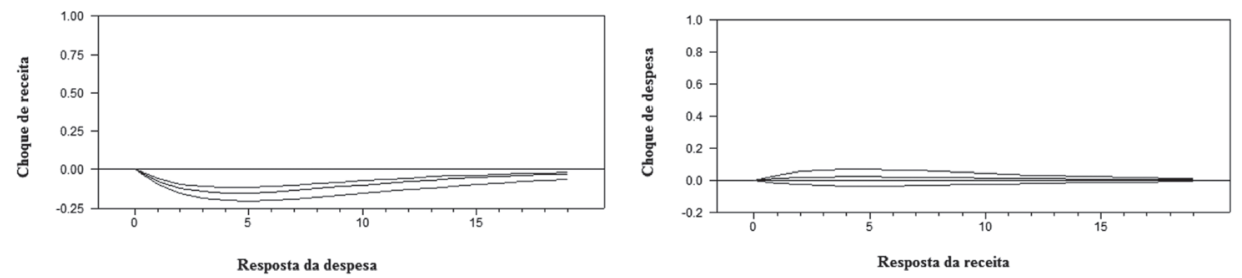

Figura 1 - Resposta da receita e dos gastos do governo central aos choques de despesa e receita governamental

Fonte: Elaboração própria.

A Figura 1 mostra as respostas da despesa e da receita governamental aos choques de receita e despesa, respectivamente, para o período de 1997 a 2015. Percebe-se que as mudanças metodológicas não alteraram os efeitos encontrados no exercício anterior, em particular, as respostas obtidas pela receita e despesa foram basicamente as mesmas. Com isso, conclui-se que, para o período de análise, o choque de despesa não trouxe efeitos significativos sobre a receita governamental, apesar de a mediana do choque ter apresentado sinal positivo. Por outro lado, o choque de receita trouxe efeitos negativos e significativos sobre as despesas governamentais, fazendo com que essas reduzam para todo o período. Esse efeito reforça o argumento já discutido anteriormente, onde a relação entre receitas e despesas governamentais no Brasil foi do tipo tax-spend.

A seguir será avaliada a importância relativa de cada variável na determinação da receita e despesa governamental por meio da decomposição da variância do erro de previsão. A Tabela 8 apresenta a decomposição da variância das séries tratadas. 
Tabela 8 - Decomposição da variância das séries de receita e despesa

\begin{tabular}{cccccccc}
\hline \multicolumn{4}{c}{ Receita } & \multicolumn{4}{c}{ Despesa } \\
\hline Período & Desvio-padrão & Receita & Despesa & Período & Desvio-padrão & Receita & Despesa \\
\hline 1 & 0.15 & 100.00 & 0.00 & 1 & 0.11 & 0.88 & 99.11 \\
$\mathbf{5}$ & 0.17 & 99.99 & 0.01 & $\mathbf{5}$ & 0.21 & 7.66 & 92.34 \\
10 & 0.17 & 99.98 & 0.02 & 10 & 0.23 & 10.45 & 89.55 \\
15 & 0.17 & 99.98 & 0.02 & 15 & 0.24 & 11.08 & 89.02 \\
$\mathbf{2 0}$ & 0.17 & 99.98 & 0.02 & $\mathbf{2 0}$ & 0.24 & 11.08 & 88.92 \\
\hline
\end{tabular}

Fonte: Elaboração própria.

Conforme se pode observar, a variabilidade da receita governamental é explicada, quase que exclusivamente, pela própria variável em todos os períodos. É bom lembrar que esse artigo analisa o comportamento fiscal do governo geral da economia brasileira até o final do ano de 2015. Assim, os anos subsequentes foram úteis para confrontar os achados desse estudo com os fatos e/ou decisões políticas tomadas. O segundo semestre do ano de 2016, por exemplo, ficou marcado pelo discurso de necessidades de reformas fiscais, direcionadas para a redução e limites as despesas do governo central. De posse dos resultados encontrados nesse artigo, essa decisão é condição suficiente para o ajuste fiscal desejado? Em outras palavras, atacar as despesas deveria ser a única medida dada pelo governo para ajustar as contas públicas? A resposta para esses questionamentos é negativa. Olhando para a decomposição da variância da série despesa é possível verificar que a receita foi responsável pela explicação $11,08 \%$ da variabilidade dessa série após 20 períodos.

Dessa forma, os dados mostram que a relação entre receitas e despesas no Brasil é do tipo tax-spend, assim, o primeiro ajuste deveria ser de elevação nas receitas, ao invés de reduções de despesas. Porém, parece difícil argumentar com a sociedade e as entidades de classes a necessidade de elevações tributárias em momentos de crise econômica, sem antes mostrar que todas as possibilidades foram adotadas. 


\section{Considerações finais}

O período que se inicia no ano de 2014 aponta para sinais claros de fragilidade fiscal, com despesas crescentes e receitas orçamentárias decrescentes. Esse cenário difere do observado até 2013, cujos testes aplicados neste artigo encontraram evidências de que o governo respeitou sua restrição orçamentária, realizando esforços para promover o equilíbrio orçamentário. Nesse sentido, esses resultados mostraram que os ajustes no longo prazo foram realizados através do sincronismo fiscal. Entretanto, no curto prazo, uma redução de impostos foi seguida por elevações nos gastos públicos. Adicionalmente, os resultados de curto prazo ofereceram, mesmo que parcialmente, suporte para a teoria da ilusão fiscal. Não sendo encontrada nenhuma evidência para as hipóteses de spend-tax e starve the beast para a economia Brasileira.

Cabe lembrar que, na segunda metade dos anos 1990, a estabilização da economia trouxe à tona os elevados déficits fiscais que tinham seus efeitos atenuados pela alta inflação. Como forma de controlar o déficit, o governo adotou uma política de austeridade fiscal com forte controle sobre o gasto público e com a criação de mecanismos que possibilitassem a elevação da receita do governo. O ápice da política de austeridade fiscal ocorreu em 1999. Dez anos depois, em 2009, os efeitos da crise financeira internacional fizeram com que o governo adotasse políticas anticíclicas para estimular a demanda agregada. No centro destas políticas estavam o aumento do gasto público e a política de incentivo ao consumo privado através da redução de impostos. Assim, a narrativa da conjuntura econômica parece consistente com os resultados deste estudo, ao negar a hipótese starve the beast e ratificar a interpretação dada pela teoria de ilusão fiscal.

Outra consideração a ser ressaltada é o papel das decisões tomadas pelo governo central, que contribuiu para o processo de fragilização fiscal do Brasil. A mudança de política econômica fez com que o governo central assumisse uma série de despesas de longo prazo, baseadas em expectativas de receitas que não se concretizaram, principalmente, as advindas da expansão dos preços das commodities de petróleo. Em decorrência disso, quando houve a reversão no ciclo econômico, as receitas caminharam na mesma direção, com tendência de queda, e as despesas seguiram sua tendência ascendente devido aos compromissos contratuais firmados em períodos anteriores. 


\section{Referências}

Baghestani, Hamid, e Mcnown, Robert. 1994. "Do Revenues Or Expenditures Respond To Budgetary Disequilibria?" Southern Economic Journal: 311-322.

Barro, Robert J. 1974. “Are Government Bonds Net Wealth?” Journal Of Political Economy: 1095-1117.

Barro, Robert J. 1979. “On The Determination Of The Public Debt.” The Journal Of Political Economy: 940-971.

Bertussi, Luís Antônio Sleimann, e Triches, Divanildo. 2012. "Multicointegração E Políticas Fiscais: Uma Avaliação De Sustentabilidade Fiscal Para Argentina, Brasil, México, Peru, Uruguai E Venezuela." Revista Economia 13 (2).

Bicalho, Aurélio. 2005. "Teste De Sustentabilidade E Ajuste Fiscal No Brasil Pós-Real." Getúlio Vargas Foundation, Unpublished Manuscript. http://bibliotecadigital.fgv.br/dspace/bitstream/handle/10438/146/2117. pdf? sequence $=1$ \&isallowed $=y$

Bierens, Herman J. 1997. "Testing The Unit Root With Drift Hypothesis Against Nonlinear Trend Stationarity, With An Application To The Us Price Level And Interest Rate.” Journal Of Econometrics 81: 29-64.

Buchanan, James M., e Wagner, Richard E. 1977. "Democracy In Deficit: The Political Legacy Of Lord Keynes.” New York.

Chan, K. 1993. "Consistency And Limiting Distribution Of The Least Squares Estimator Of A Threshold Autoregressive Model." Annals Of Statistics 21: 520-533.

Cuñado, Juncal, Gil-Alana, Luis Alberiko e Fernando Pérez De Gracia. 2004. "Is The US Fiscal Deficit Sustainable?: A Fractionally Integrated Approach.” Journal Of Economics And Business 56 (6): 501-526.

Enders, Walter, e Siklos, Pierre L. 2001. "Cointegration And Threshold Adjustment." Journal Of Business \& Economic Statistics 19 (2): 166-176.

Engle, Robert F., e Granger, Clive Wj. 1987. "Co-Integration And Error Correction: Representation, Estimation, And Testing." Econometrica: Journal Of The Econometric Society: 251-276.

Friedman, Milton. 1978. “The Limitations Of Tax Limitation.” Quadrant 22 (8): 22.

Friedman, Milton. 2003. "What Every American Wants.” Wall Street Journal. New York January 15.

Gadelha, Sérgio R. 2011. "Causalidade Temporal Entre Receita E Despesa Governamentais.” Análise Econômica 29 (56).

Gadelha, Sérgio R. De Brito, e Divino, José Angelo. 2013. “Uma Análise Da Ciclicidade Da Política Fiscal Brasileira." Estudos Econômicos 43(4).

Granger, Clive WJ. 1969. "Investigating Causal Relations By Econometric Models And Cross-Spectral Methods." Econometrica: Journal Of The Econometric Society: 424-438.

Granger, C. WJ. 1988. "Some Recent Development In A Concept Of Causality.” Journal Of Econometrics 39 (1-2): 199-211.

Gregory, Allan W., e Bruce E. Hansen. 1996. "Residual-Based Tests For Cointegration In Models With Regime Shifts.” Journal Of Econometrics 70: 99-126.

Hondroyiannis, George, e Papapetrou, Evangelia. 1996. "An Examination Of The Causal Relationship Between Government Spending And Revenue: A Cointegration Analysis.” Public Choice 89 (3-4): 363-374.

Hakkio, Craig S., e Rush, Mark. 1991. "Is The Budget Deficit “Too Large?” Economic Inquiry 29 (3): 429-445. Johansen, Soren. 1995. "Likelihood-Based Inference In Cointegrated Vector Autoregressive Models." OUP Catalogue.

Linhares, Fabricio Carneiro, Simonassi, Andrei Gomes, Nojosa, Glauber Marques. 2012. "A Dinâmica Do Equilíbrio Financeiro Municipal E A Lei De Responsabilidade Fiscal.” Economia 13 (3b). 
Mattos, Enlinson, e Rocha, Fabiana. 2001. “Correção Monetária E O Equilíbrio Do Orçamento.” Pesquisa e Planejamento Econômico 31 (2).

Mello, Luiz. 2005. “Estimating A Fiscal Reaction Function.” OECD Economics Department Working Papers 423.

Meltzer, Allan H., e Richard, Scott F. 1981. "A Rational Theory Of The Size Of Government.” Journal Of Political Economy 89 (5).

Niskanen, William A. 2006. "Limiting Government: The Failure Of Starve The Beast.” Cato J. 26: 553.

Nobrega, W. C. L. 2016. "Interação Entre A Política Fiscal E Monetária: Uma Análise Sobre O Regime De Dominância Vigente Na Economia Brasileira.” Dissertação De Mestrado. Universidade Federal Da Paraíba.

Owoye, Oluwole, Onafowora, Olugbenga A. 2011. "The Relationship Between Tax Revenues And Government Expenditures In European Union And Non-European Union OECD Countries.” Public Finance Review 39 (3): 429-461.

Paleologou, Suzanna-Maria. 2013. “Asymmetries In The Revenue-Expenditure Nexus: A Tale Of Three Countries." Economic Modelling 30: 52-60.

Pastore, A., Gazzano, M., Pinotti, M. 2014. Inflação E Crises: O Papel Da Moeda. 1․ Ed. Elsevier Brasil.

Payne, James E. 2003. “A Survey Of The International Empirical Evidence On The Tax-Spend Debate.” Public Finance Review 31 (3): 302-324.

Peres, Marco Aurélio Ferreira, e Ellery, JR, R. G. E. 2009. “Efeitos Dinâmicos Dos Choques Fiscais Do Governo Central No PIB Do Brasil.” Pesquisa E Planejamento Econômico 39 (2).

Phillips P. C. B., e Ouliaris, S. 1990. "Asymptotic Properties Of Residual Based Tests For Cointegration." Econometrica 58 (1): 165-193.

Ricardo, David. 1821. "Essay On The Funding System.” Encyclopaedia Britannica.

Romer, Christina D., e Romer, David H. 2009. "Do Tax Cuts Starve The Beast? The Effect Of Tax Changes On Government Spending.” Brookings Papers On Economic Activity 1.

Saunoris, James W., e Payne, James E. 2010. "Tax More Or Spend Less? Asymmetries In The UK Revenue-Expenditure Nexus.” Journal Of Policy Modeling 32 (4): 478-487.

Silva, Cleomar Gomes da, Machado, Sérgio Jurandyr, Lopes, Denílson Torcate, e André Marques Rebelo. 2010. "Receitas E Gastos Governamentais: Uma Análise De Causalidade Para O Caso Brasileiro." Economia Aplicada 14 (4): 265-275.

Schettini, Bernardo P. 2012. “Análises Da Dinâmica Orçamentária Dos Municípios Brasileiros: Uma Aplicação Da Metodologia VAR Com Dados Empilhados.” Economia Aplicada 16 (2): 291-310.

Wagner, Richard. 1976. "Revenue Structure, Fiscal Illusion And Budgetary Choice.” Public

Choice 25: 45-61.

Young, Andrew T. 2009. “Tax-Spend Or Fiscal Illusion.” Cato J. 29: 469. 